\title{
Serotype transmission dynamics and reduced incidence of invasive pneumococcal disease caused by different serotypes after implementation of non-pharmaceutical interventions during COVID-19 pandemic
}

\begin{abstract}
To the Editor:
Copyright @The authors 2021

This version is distributed under the terms of the Creative Commons Attribution NonCommercial Licence 4.0. For commercial reproduction rights and permissions contact permissions@ersnet.org

Received: 2 April 2021 Accepted: 7 July 2021

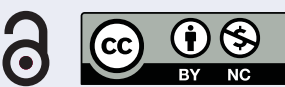

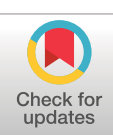

Shareable abstract (@ERSpublications)

Significant reduction in the incidence rates of both vaccine and non-vaccine serotype invasive pneumococcal disease among children and adults during the COVID-19 pandemic were observed after the implementation of non-pharmaceutical interventions https://bit.ly/36CAute

Cite this article as: Janapatla RP, Chen C-L, Dudek A, et al. Serotype transmission dynamics and reduced incidence of invasive pneumococcal disease caused by different serotypes after implementation of non-pharmaceutical interventions during COVID-19 pandemic. Eur Respir J 2021; 58: 2100978 [DOI: 10.1183/13993003.00978-2021].

Prior to 2020, IPD incidence rates followed a seasonal pattern (figure 1a); however, from August to December 2020 incidence rates were plateaued around 0.068 per 100000 per month. A total of 285 IPD cases were detected from January 2020 to February 2021 and the monthly incidence rates significantly decreased between 37\% and 77\% $(p<0.001-0.05)$ from February 2020 to January 2021, when compared to the corresponding month in the previous year, except in June when the incidence is usually lower (figure 1a). 3 years prior to the COVID-19 pandemic (2017-2019), annual IPD incidence rates were highly stable (1.913-1.932 per 100000), but reduced significantly from 1.932 per $100000(n=456)$ in $2019(95 \%$ CI $0.127-0.195$ per 100000 per month) to 0.972 per $100000(n=229)$ in 2020 (95\% CI 0.038-0.124 per 100000 per month) ( $\mathrm{p}=0.001$ ), corresponding to a $49.7 \%$ reduction (figure $1 \mathrm{a}$ ).
\end{abstract}



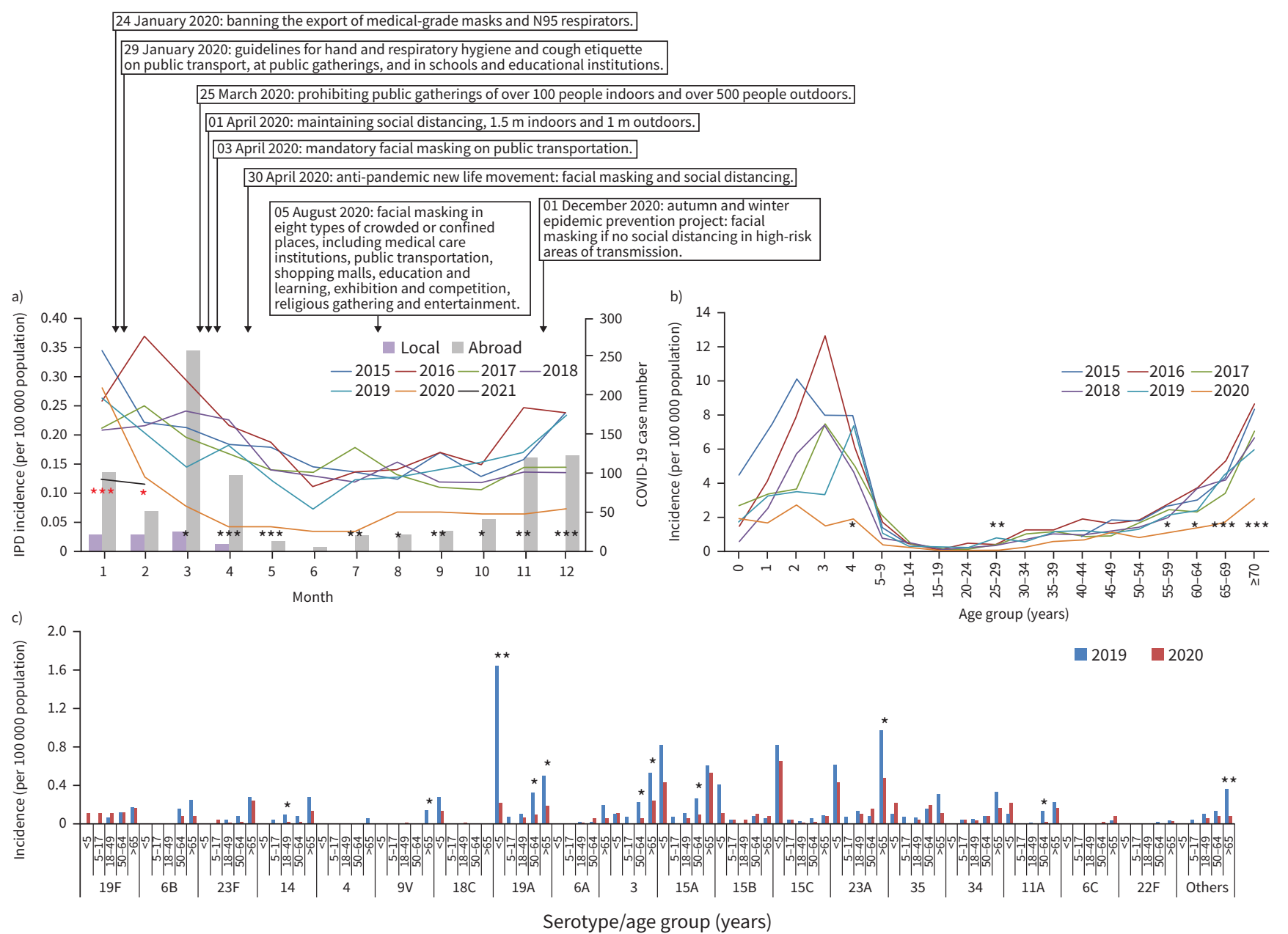

FIGURE 1 a) Monthly incidence rates of invasive pneumococcal disease (IPD) during 2015-2020 and monthly confirmed cases (local and imported) of coronavirus disease 2019 (COVID-19) between January 2020 and February 2021 in Taiwan. Arrows indicate timeline of non-pharmaceutical interventions implemented. Asterisks indicate significant differences in incidence rate at each month; black asterisks indicate differences between months in 2019 versus the same month in 2020, and red asterisks 2020 versus 2021. b) Changes in the estimated annual incidence rates of IPD in different age groups during 2015-2020. c) Changes in age group-specific incidence of IPD caused by different serotypes during $2019-2020$. ${ }^{\star}: p<0.05,{ }^{\star \star}: p \leqslant 0.01 ;{ }^{* \star *}: p \leqslant 0.001$.

Notably, significant reduction (40-67\%; $\mathrm{p}<0.001-0.05$ ) in IPD incidence rates was observed across all age groups in 2020 (figure 1b). Among the age groups at high risk for IPD, the incidence reduced significantly among children aged $<5$ years by $46.1 \%$ (from 4.61 to 2.49 per 100000 ; $\mathrm{p}=0.014$ ) and in older adults (>65 years) by $47.0 \%$ (from 5.38 to 2.85 per 100000 ; $\mathrm{p}<0.001$ ). In high pneumococcal transmission settings like schools, among the primary and secondary school aged children (6-17 years) with mandatory masking when attending classes, IPD cases decreased by $65.5 \%$ (from 0.55 to 0.19 per $100000 ; \mathrm{p}<0.027)$.

Among children aged $<5$ years (figure $1 \mathrm{~b}$ ), serotype 19A IPD incidence rate declined remarkably by $86.6 \%$ to 0.22 per 100000 ( $n=2, p=0.003)$. IPD caused by non-PCV13 serotypes (15A, 15B, 15C and 23A) also decreased moderately by $27.0 \%$, from 2.87 to 2.05 per 100000 , but the decrease was not statistically significant $(\mathrm{p}=0.258$ ) (figure $1 \mathrm{~b}$ ). Further analysis revealed that among older adults ( $>65$ years) with highest incidence rate, IPD caused by PCV13 serotypes declined by 54.6\% (from 2.38 to 1.08 per 100000; $\mathrm{p}<0.001$ ) and non-PCV13 serotypes by $40.8 \%$ (from 2.99 to 1.77 per 100000 ; $\mathrm{p}<0.001$ ) (figure $1 \mathrm{c}$ ). Figure 1c also shows the change in individual serotypes. There were three findings: significant reduction in 19A IPD in both children aged $<5$ years and adults $>50$ years; significant reduction in serotype 3 IPD only in the adults aged $>50$ years; and significant reduction of IPD caused by non-vaccine serotypes $11 \mathrm{~A}, 15 \mathrm{~A}$, 23A and others (serogroups 6, 15, 16 and serotypes 7F, 10A) only in the adults and elderly population. 
After the first case of COVID-19 was reported on 21 January, 2020, the monthly incidence of IPD significantly decreased from February 2020 to January 2021, indicating that significant IPD incidence rate reduction can be seen within a month after the implementation of NPIs to control COVID-19 (figure 1c). Subsequently, we observed incidence of IPD among children $<5$ years (mostly attending day care centres) declined substantially by $86.6 \%$ in highly invasive serotype 19A IPD and a modest reduction of $39.1 \%$ in IPD caused by non-PCV13 serotypes (serogroups 15 and 23A) that emerged in the post-PCV13 era. Because these non-PCV13 serotypes tended to express less invasive potential, the reduction of IPD by these non-PCV13 serotypes was not as prominent as that by 19A. The result suggests that among the vaccinated children, basic COVID-19 control measures can accelerate the elimination of the residual IPD by vaccine serotypes, such as 19A, and simultaneously assist in reducing the transmission of the predominant non-vaccine serotypes. On the other hand, reduction in IPD caused by non-vaccine serotypes 11A, 15A and 23A was more prominent in the adults or elderly, suggesting these non-PCV13 serotypes may be more virulent to the elderly. Another possibility is that these non-vaccine serotypes are mainly carried by children, rather than adults.

The effectiveness of NPIs in a high transmission setting was further evident by near elimination of IPD (only five cases) in 2020 among the school aged children (6-17 years) who were recommended to follow mandatory masking policy when attending classes. The use of facemasks in adult caregivers in day care centres, kindergartens and schools might have reduced the risk of transmission of invasive serotypes to children, thereby reducing IPD. To control the spread of COVID-19, almost all countries with robust healthcare systems have ordered closure of schools at some point of time for a specific duration in 2020, but did not happen in Taiwan during the study period. Winter break was extended by two weeks (11 to 25 February, 2020) for schools as an initial response to reduce the risk of COVID-19 clusters. However, it would be interesting to see IPD surveillance data from countries such as Sweden where facial masks were less used, COVID-19 control measures were less strict, and junior high schools remained open [11].

Among older adults who tended to follow COVID-19 control measures more strictly, IPD incidence rate decreased by $47 \%$, including a significant reduction in IPD due to serotypes 3, 19A and 23A. Although the reduction could be partly linked to indirect effect on IPD in adults after the childhood PCV13 vaccination and 23-valent polysaccharide vaccine (PPV23) use for adults aged $\geqslant 75$ years [10], such a dramatic reduction within 1 year apparently was related to the NPIs deployed in the pandemic era. In a recent study we failed to detect serotype 3 by culture and PCR from the nasopharynx of children; however, we observed elimination of serotype 3 IPD cases in children ( $<5$ years) and, in contrast, the continued prevalence of serotype 3 IPD in older adults (>65 years), meaning that adults are potential reservoirs for serotype 3 from where adult pneumococcal infections and transmissions to their close contacts can occur [10, 12].

NPIs that were initiated by authorities in January 2020 to reduce transmission of SARS-COV-2 in crowded or enclosed places were recommended throughout the study period. Regular press conferences were held by Taiwan CDC to announce updated policies and measures for residents and competent authorities. Most strikingly, during the study period Taiwan did not impose mandatory stay-at-home orders for residents or lockdowns on cities and regions. In England it was noted that IPD incidence in 2019-2020 was 30\% lower, compared to 2018-2019, and IPD incidence rates were found to decline substantially across all age groups during the period March-June 2020 [13], which could be linked to strict lockdowns. Yun et al. [14] reported a 22\% decline in IPD incidence in South Korea where severe lockdowns were not implemented, but major outbreaks occurred in cities such as Daegu and Seoul. Recently, GAO et al. [15] reported from Taiwan that the adjusted pneumonia and influenza mortality rate in 2020 was significantly lower than that in 2019. Since nasopharyngeal colonisation with pneumococci precedes IPD, the main limitation of this study was that the nasopharyngeal carriage status in children and adults was not determined during the pandemic.

In conclusion, the study provides us an opportunity to explore the differential serotype transmission dynamics and their invasive potentials among children and adults. Significant reduction in both vaccine and non-vaccine serotype IPD incidence rates among children and adults during the COVID-19 pandemic supports the continued implementation of basic NPIs, such as facial masking, enhanced hand hygiene and social distancing, at least in high transmission risk settings, to reduce viral and bacterial respiratory tract infections, including IPD.

Rajendra Prasad Janapatla $\odot^{1}$, Chyi-Liang Chen $\odot^{1}$, Anna Dudek ${ }^{1}$, Hsin-Chieh Li $^{1}$, Hsin-Ping Yang ${ }^{1}$, Lin-Hui $\mathrm{Su}^{1}$ and Cheng-Hsun Chiu ${ }^{1,2}$

${ }^{1}$ Molecular Infectious Disease Research Center, Chang Gung Memorial Hospital, Chang Gung University College of Medicine, Taoyuan, Taiwan. ${ }^{2}$ Division of Pediatric Infectious Diseases, Dept of 
Pediatrics, Chang Gung Memorial Hospital, Chang Gung University College of Medicine, Taoyuan, Taiwan.

Corresponding author: Cheng-Hsun Chiu (chchiu@adm.cgmh.org.tw)

Acknowledgements: We thank Wei-Ju Su, Division of Acute Infectious Disease, Centers for Disease Control, Ministry of Health and Welfare, Taiwan for assistance in data collection.

Conflict of interest: None declared.

Support statement: This study was supported by grants from Ministry of Science and Technology (MOST109-2327-B-182-002) and Chang Gung Memorial Hospital (CMRPG3H0041-3 and CORPG3K0311), Taiwan. Funding information for this article has been deposited with the Crossref Funder Registry.

\section{References}

1 Wang CJ, Ng CY, Brook RH. Response to COVID-19 in Taiwan: big data analytics, new technology, and proactive testing. JAMA 2020; 323: 1341-1342.

2 Askitas N, Tatsiramos K, Verheyden B. Estimating worldwide effects of non-pharmaceutical interventions on COVID-19 incidence and population mobility patterns using a multiple-event study. Sci Rep 2021; 11: 1972.

3 Cheng HY, Li SY, Yang CH. Initial rapid and proactive response for the COVID-19 outbreak - Taiwan's experience. J Formos Med Assoc 2020; 119: 771-773.

4 Chuang YT, Liu CE. Who wears a mask? Gender differences in risk behaviors in the COVID-19 early days in Taiwan. Economics Bulletin 2020; 40: 2619-2627.

5 Soo RJJ, Chiew CJ, Ma S, et al. Decreased influenza incidence under COVID-19 control measures, Singapore. Emerg Infect Dis 2020; 26: 1933-1935.

6 Kuo SC, Shih SM, Chien LH, et al. Collateral benefit of COVID-19 control measures on influenza activity, Taiwan. Emerg Infect Dis 2020; 26: 1928-1930.

7 Galvin CJ, Li YJ, Malwade S, et al. COVID-19 preventive measures showing an unintended decline in infectious diseases in Taiwan. Int J Infect Dis 2020; 98: 18-20.

$8 \mathrm{Lim} \mathrm{RH}$, Chow A, Ho HJ. Decline in pneumococcal disease incidence in the time of COVID-19 in Singapore. J Infect 2020; 81: e19-e21.

9 Partridge E, McCleery E, Cheema R, et al. Evaluation of seasonal respiratory virus activity before and after the statewide COVID-19 shelter-in-place order in northern California. JAMA Netw Open 2021; 4: e2035281.

10 Lu CY, Chiang CS, Chiu CH, et al. Successful control of Streptococcus pneumoniae 19A replacement with a Catch-up Primary Vaccination Program in Taiwan. Clin Infect Dis 2019; 69: 1581-1587.

11 Vlachos J, Hertegård E, Svaleryd HB. The effects of school closures on SARS-CoV-2 among parents and teachers. Proc Natl Acad Sci USA 2021; 118: e2020834118.

12 Janapatla RP, Hsu MH, Chen $\mathrm{CL}$, et al. Persistence of immunity in children immunised with 13-valent pneumococcal conjugate vaccine and impact on nasopharyngeal carriage: a cross-sectional study. Thorax 2020; 75: 689-692.

13 Amin-Chowdhury Z, Aiano F, Mensah A, et al. Impact of the coronavirus disease 2019 (COVID-19) pandemic on invasive pneumococcal disease and risk of pneumococcal coinfection with severe acute respiratory syndrome coronavirus 2 (SARS-CoV-2): prospective national cohort study, England. Clin Infect Dis 2021; 72: e65-e75.

14 Yun HE, Ryu BY, Choe YJ. Impact of social distancing on incidence of vaccine-preventable diseases, South Korea. J Med Virol 2021; 93: 1814-1816.

15 Gao W, Sanna M, Huang G, et al. Examining population health during the COVID-19 pandemic: all-cause, pneumonia and influenza, and road traffic deaths in Taiwan. Ann Intern Med 2021: 174: 880-882. 